Psychotherapeut 2011 · 56:466-467

DOI 10.1007/s00278-011-0872-x

Online publiziert: 10. November 2011

(c) Springer-Verlag 2011

\author{
Harald J. Freyberger ${ }^{1} \cdot$ Wolfgang Schneider $^{2}$ \\ ${ }^{1}$ Klinik und Poliklinik für Psychiatrie und Psychotherapie, \\ Universitätsmedizin Greifswald \\ ${ }^{2}$ Klinik und Poliklinik für Psychosomatische Medizin und Psychotherapie, \\ Universität Rostock
}

\title{
Einfluss des Internet auf die Psychotherapie
}

zung, sodass sich neue Behandlungsformen entwickeln.

mit einer sich stetig ausweitenden Nutzung des Internet einem Prozessgeschehen gegenüber, das viele Bereiche des menschlichen Lebens verändert und auch breit in persönliche Lebensabläufe eingegriffen hat. Information, Kommunikation und zwischenmenschliche Kontakte haben durch die Eigenschaften des Internet, wie den leichten Zugang, die dort zumindest teilweise akzeptierte Anonymität, die niedrigen Kosten und die weltweite Vernetzung neue Möglichkeiten und Risiken ans Tageslicht gebracht, die selbstverständlich auch die Psychotherapie nicht ausklammern. Für viele Psychotherapeuten und Psychotherapieforscher, die Beziehung und Bindung als zentrales Paradigma psychotherapeutischer Wirkung und Wirksamkeit ansehen, ist die Perspektive einer Internetpsychotherapie mit unliebsamen Assoziationen verknüpft. Christiane Eichenberg (Köln) stellt in ihrer Übersichtsarbeit neben dieser Ebene der Veränderung traditioneller therapeutischer Beziehungen noch 4 weitere Schnittstellen zwischen Internet und Psychotherapie heraus:

1. Das Internet liefert eine Fülle von gesundheitsrelevanten Informationen, die für die Nutzer als Ressource oder - bei unzureichender Qualität - als nachteilig verstanden werden können.

2. Als Kommunikationsmedium kann das Internet therapeutische Interventionen unterstützen, wie etwa durch psychologische Onlineberatung.

3. Die Internetnutzung ist mit klinisch relevanten Problemen assoziiert, wie etwa der exzessiven suchtartigen Nut-
4. Das Internet wird inzwischen für die Psychotherapieforschung genutzt.

Für die Ebene der gesundheitsrelevanten Informationen ist u. a. zu berücksichtigen, dass Psychoedukation und die Wissensakquisition zu psychischen Störungen bereits für sich genommen mit bemerkenswerten Effektstärken verknüpft sind und insbesondere in Flächenländern und für die in ihrer Mobilität eingeschränkten Nutzergruppen in der Zukunft von wachsender Bedeutung sein dürften. Wie Andreas Hill (Hamburg) in seiner Übersichtsarbeit zur Sexualität in Zeiten des Internet herausarbeitet, haben die Ebene der Vermittlung gesundheitsrelevanter Informationen und die Ebene der Kommunikation noch eine weitere Dimension v. a. für benachteiligte und Randgruppen: $\mathrm{Zu}$ den kaum untersuchten Chancen des Internet zählen nämlich die Erleichterung sexueller und sozialer Kontakte, besonders für Menschen mit sozialen Schwierigkeiten, für sexuelle Minderheiten (z. B. Schwule, Lesben, Transgender, Sadomasochisten, Fetischisten), der Abbau von Vorurteilen und Stereotypen, eine bessere Abstimmung von Interessen bei der Partnerwahl und die zahlreichen, auch seriösen Informationen und Beratungsangebote $\mathrm{zu}$ sexuellen Themen. Diese Aspekte lassen sich zumindest teilweise auch auf andere Bereiche menschlichen Lebens und andere sozial potenziell benachteiligte Gruppen übertragen. Umgekehrt zeigt Hill und diese dialektische Betrachtungsweise erscheint uns bei allen Chancen und Risi- ken des Internet angemessen -, dass wir in der klinischen Praxis häufig mit den problematischen Auswirkungen der Internetnutzung konfrontiert werden. Er nennt im Kontext der Sexualität Internetpornografie und Cybersex, v. a. die Vermeidung von "realen“ - sexuellen und nichtsexuellen - zwischenmenschlichen Kontakten, die Belastung von Partnerschaften (z. B. durch „Cyberuntreue“), die Verstärkung von selbst- oder fremdschädigenden Fantasien und Impulsen bis hin zu strafrechtlich relevantem Verhalten, wie dem Konsum und der Verbreitung von Kinderpornografie.

Die Nutzung des Internet ist also auch mit Risiken verknüpft, die allerdings realistisch eingeschätzt werden sollten. So hat eine kürzlich veröffentlichte repräsentative Allgemeinbevölkerungsuntersuchung gezeigt, dass die Prävalenz der Internetabhängigkeit in Abhängigkeit von dem diagnostischen „Cut-off“-Wert zwischen 1 und 1,5\% liegt (Rumpf et al. 2011). In diesem Heft von Psychotherapeut beschäftigen sich Christiane Eichenberg, Andrea Flümann und Kristin Hensges (Köln) in einer empirischen Nutzerbefragung mit den sog. Pro-Ana-Foren im Internet, den auch in der Öffentlichkeit $z$. T. äußerst kritisch diskutierten Internetforen, insbesondere junger essgestörter Frauen. Ihre Ergebnisse untermauern in keiner Weise Forderungen nach einer Schließung derartiger Foren, wie sie in der Vergangenheit öffentlich diskutiert wurden.

In der Psychotherapieforschung bzw. im Bereich psychotherapeutischer Interventionen wird das Internet sehr verschiedenartig genutzt. Neben psychotherapeu- 
tischen Behandlungsansätzen im engeren Sinne, wie narrativen Therapieansätzen (etwa "testimonial therapy“; z. B. Böttche et al. 2011; www.lebenstagebuch.de), werden derzeit in Deutschland in breitem Umfang internetbasierte Interventionen zu Vor- und Nachbereitung ambulanter oder stationärer psychotherapeutischer Behandlungen untersucht. In diesem Kontext stellen Benjamin Zimmer, Christian Peter Dogs und Hans Kordy (Heidelberg/Scheidegg) die Ergebnisse einer randomisierten, kontrollierten Studie vor, die sich mit einer internetbasierten Vorbereitung auf eine stationäre psychosomatischpsychotherapeutische Behandlung beschäftigt. Ina Beintner und Corinna Jacobi (Dresden) stellen zudem ein von ihnen entwickeltes Behandlungsmanual für die internetgestützte Nachsorge bei Bulimia nervosa, einschließlich eines dazugehörigen Studiendesigns, vor.

Stephanie Bauer, Eberhard Okon und Rolf Meermann (Heidelberg, Bad Pyrmont) beschäftigen sich mit einem Ansatz der SMS-basierten Nachsorge von zuvor psychotherapeutisch behandelten Patienten, der derzeit auch von weiteren Arbeitsgruppen verfolgt wird (z. B. van den Berg et al. 2011). In ihrer empirischen Studie können sie die Wirksamkeit eines SMS-basierten Programms zur Nachsorge bei stationärer Psychotherapie von Essstörungen belegen. In diesem Kontext muss auch die Übersichtsarbeit von Markus Wolf gelesen werden, der sich mit supportivem Monitoring in der Psychotherapie beschäftigt.

Es sollte nicht vergessen werden, dass die ersten Ansätze internetbasierter, im weitesten Sinne psychotherapeutischer Interventionen in epidemiologischen Versorgungsstudien in den 1980er Jahren entwickelt wurden und dass sich daraus jetzt zunehmend Interventionsansätze entwickeln, die wahrscheinlich in wenigen Jahren Eingang in die klinische Versorgungspraxis finden werden.

\section{Korrespondenzadresse}

\section{Prof. Dr. Harald J. Freyberger}

Klinik und Poliklinik für Psychiatrie und

Psychotherapie, Universitätsmedizin Greifswald

Ellernholtzstr. 1-2, 17487 Greifswald

freyberg@uni-greifswald.de

\section{Literatur}

Böttche M, Kuwert P, Knaevelsrud C (2011) Posttraumatic stress disorder in older adults: an overview of characteristics and treatment approaches. Int J Geriatr Psychiatry. doi: 10.1002/gps.2725

Rumpf HJ, Meyer C, Kreuzer A, John U (2011) Prävalenz der Internetabhängigkeit (PINTA). Bericht an das Bundesministerium für Gesundheit. http://drogenbeauftragte.de/ fileadmin/dateien-dba/DrogenundSucht/Computerspiele_Internetsucht/ Downloads/PINTA-Bericht-Endfassung_280611. pdf

van den Berg N, Grabe HJ, Freyberger HJ, Hoffmann W (2011) A telephone- and text-message based telemedical care concept for patients with mental health disorders - study protocol for a randomized, controlled study design. BMC Psychiatry 11:30
Schwerpunktthemen

Das Herausgebergremium der Zeitschrift Psychotherapeut lädt Autorinnen und Autoren ein, an den geplanten Schwerpunkten mitzuarbeiten und geeignete Manuskripte einzureichen. Diese werden dem üblichen Reviewverfahren unterzogen. Darüber hinaus freuen wir uns über die Zusendung freier (unaufgeforderter) Originalia zu selbstgewählten Themen.

Bitte schicken Sie Ihren Beitrag an die Redaktion:

Regine.Karcher-Reiners@springer.com

Die Schwerpunktplanung ist vorläufig und kann kurzfristig umdisponiert werden.

Heft 3/12: Neurowissenschaftliche Befunde der Psychotherapie (Deadline 14.1.2012)

Heft 4/12: Psychoedukation (Deadline 28.2.2012)

Heft 5/12: Negativwirkungen und Folgen von Psychotherapie (Deadline 30.4.2012)

Heft 6/12: Psychosenpsychotherapie (Deadline 30.6.2012) 